\title{
CoviD-19 Remodelling elective hospital services in the COVID-19 era - designing the new normal
}

\author{
Authors: Lamin A King ${ }^{A}$ and Sandeep S Hothi ${ }^{B}$
}

The provision of elective clinical services has decreased during the initial phase of the coronavirus disease 2019 (COVID-19) pandemic to enable hospitals to focus on acute illness. Any end to the pandemic through widespread vaccination, effective treatment or development of herd immunity may be years away. Until then, hospitals will need to resume treating other diseases while also attempting to eradicate transmission of COVID-19 within the healthcare setting. In this article we suggest six major themes which could affect the design and delivery of elective clinical services: hospital avoidance, separation of high- and low-risk groups, screening, maintenance of adequate infection control, and new ways of working.

KEYWORDS: Healthcare system design, COVID-19, modernising healthcare

DOI: $10.7861 /$ fhj.2020-0079

\section{Introduction}

The provision of elective clinical services decreased during the initial phase of the coronavirus disease 2019 (COVID-19) pandemic. ${ }^{1,2}$ There was a diversion of resources to rapidly increase capacity for emergency and critical care, and specific measures were implemented to protect those vulnerable to poor outcomes from COVID-19. Any end to the pandemic through widespread vaccination, effective treatment or development of herd immunity may be years away. ${ }^{2,3}$ Until then, hospitals will need to resume treating other diseases while also attempting to eradicate transmission of COVID-19 within the healthcare setting. This will involve more than simply returning to doing the usual things the usual way.

In this article we suggest six major themes which could affect the design and delivery of elective clinical services: hospital avoidance, separation of high and low-risk groups, pre-admission screening, maintenance of adequate infection control, and the introduction of alternative medical practices and new ways of working.

\section{Hospital avoidance}

The widespread use of telephone or video clinics has enabled many elective consultations to continue during the pandemic

Authors: Aconsultant in acute medicine, Dorset County Hospital, Dorchester, UK; ${ }^{B}$ Consultant cardiologist, The Royal Wolverhampton NHS Trust, Wolverhampton, UK while protecting vulnerable patients from the risks of travel and hospital attendance. Until hospitals can be confidently excluded as a nosocomial reservoir for COVID-19, ${ }^{3}$ such consultations should remain the default unless face-to-face contact is clinically required, for instance, for physical examination where this is likely to alter diagnosis or management. Nearly all of the usual clinical and administrative elements of an in-person assessment are involved and it is reasonable that electronic consultations are scheduled to take equivalent time and are billed at equivalent rates.

Medical culture traditionally requires in-person patient attendance to obtain written consent for procedures, though in some specialties and organisations postal consent is permitted. Remote consultations should be used to obtain informed consent as far as possible and written confirmation could be obtained with a digital consent form.

Where patients must attend in person, they should attend alone unless it is absolutely necessary that they are accompanied. Of course, in certain situations moral support from a friend or relative is desirable and this could be permitted in exceptional situations, while in more routine cases they could join the consultations by electronic conferencing.

Furthermore, we must consider how other areas of care such as physiotherapy, psychiatric care, and cardiac rehabilitation may be adapted for remote delivery. These could be delivered using a hybrid model where specialist opinion is provided to the patient and their local doctor to limit the need to travel to the hospital setting. Where face-to-face clinical examination is deemed essential this could either be performed locally or in hospital if beyond the skillset of the local clinician.

Thus, where face-to-face assessment or care is required, consideration must be given to whether it could occur either at home or at a decentralised location within the community. Innovative scheduling will be required to limit staff mixing community and in-hospital duties. This may inevitably be more resource-intensive than previous models of centralised patient care.

\section{Patient separation}

Waiting rooms and recovery areas help to optimise operational efficiency in rate-limiting areas of service delivery as well as in other critical hospital services such as pharmacy and patient transport. Any implementation of social distancing measures (such as removing alternate seats) will reduce capacity within these areas, leading to services running at sub-maximal capacity. Innovative measures will be required, such as patients waiting in their cars before being called to waiting rooms. Drive-through services, 
where possible, represent another possible solution, and might be applicable for collection of prescription medication and pacemaker checks.

Thought must be given to the flow of patients through hospital buildings to avoid vulnerable and infected patients sharing common areas. ${ }^{4}$ The use of separate entrances should be considered, and the relocation of certain services or departments may be required. Separate services may be needed for inpatients and outpatients, with some services potentially delivered on different days, at quieter times, on separate sites, at increased intervals or with fewer staff.

Where adequate separation is not possible, then novel solutions must be considered including pooling of services and sharing of locations between hospitals or partnerships with private hospitals.

\section{Pre-admission screening for COVID-19}

Although relatively basic, temperature checks and symptom questionnaires have an important role in the screening of patients prior to elective procedures. However, patients may have a temperature or symptoms for reasons other than COVID-19 and conversely these checks may fail to identify those in the pre-symptomatic phase of COVID-19 and those remaining asymptomatic or oligosymptomatic throughout their illness. ${ }^{5}$

Consequently, widespread antigen testing (currently comprising nasopharyngeal and oropharyngeal swabs to provide test material for quantitative PCR) will be necessary to determine the presence or absence of active infection. Results must be rapid enough to efficiently inform pathways for patient care. Concerns about test sensitivity will require advancements in both the testing method and specimen collection. Thus, given the insufficiently sensitive antigen testing currently available, it is the positive result that is most informative, while a negative result should still be treated with high-level PPE and appropriate patient separation in place.

The role of pre-admission serological testing remains unclear but might supplement antigen testing, particularly when the latter is negative. It may take as long as 20 days post-infection for antibodies to become detectable. ${ }^{6,7}$ Furthermore, positive serology may indicate historic, recent or active infection. Antibodies are not known to indicate immunity to COVID-19. Even those with immunity to infection might still be vectors for passive transmission and deviation from standard infection control measures - hand washing and use of appropriate level of PPE - should therefore not be omitted on the basis of negative serological testing.

While the combination of antigenic and serological screening is essential, all screening modalities have their drawbacks. Consequently, preparatory self-isolation for 14 days prior to attendance for elective procedures and certain diagnostic tests will be required to reduce the chance of an infectious patient attending prior to the development of symptoms. ${ }^{8}$ Elective faceto-face meetings vary in their risk of COVID-19 transmission, and all but those associated with the lowest risk should require selfisolation, and all cases should have antigen testing.

\section{Infection control}

Although healthcare systems have risen to the challenge of providing emergency and critical care to patients with COVID-19 alongside those who are not infected, contentious issues have included limited availability of PPE within the health and social care setting, the provision of PPE appropriate to clinical risk, and inadequate availability of antigenic and serological testing. It is against this background that elective services must be introduced with the intention of eradicating in-hospital transmission of COVID-19.

A resumption of elective services will place additional demand on PPE for patients and staff and must be planned carefully. Nevertheless, adequate levels of PPE appropriate to risk must be in place to enable resumption of elective activity that is safe for patients and staff. Increased requirements for cleaning of clinical areas will lengthen the interval between elective consultations, scans and procedures. There is also a need to consider the safety of staff deemed at higher risk from COVID-19 on the basis of age, ethnicity, medical history or other factors.

Essential measures such as strict segregation of COVID and nonCOVID areas within wards may reduce capacity and limit scope for provision of elective inpatient services. Overcoming this will require significant consideration and innovation and possibly the development of new infrastructure.

The high rates of infection and overall excess mortality seen in care homes, and high rates of infection observed amongst hospital staff, support the theory that COVID-19 may now be a nosocomial infection centred in institutional settings, although this will benefit from more widespread testing. ${ }^{3}$

While a nosocomial phase of COVID-19 brings additional challenges, adopting the simultaneous aims of reintroducing elective medical services and eradicating in-hospital infection may offer the opportunity to largely eradicate the disease within the UK to the levels seen elsewhere through reduction of the in-hospital reproduction ( $R$ ) value, in advance of availability of a vaccine or specific antiviral treatment. ${ }^{9-11}$ Staff and patients must be well resourced and must remain meticulous in all aspects of infection control. Frequent testing of all staff, including asymptomatic staff, to identify and isolate all active carriers may reduce the spread of infection within healthcare facilities given the high prevalence of asymptomatic staff in such organisations. ${ }^{12}$

Face masks for patients and staff in clinical and even nonclinical settings should be considered, as although they may not significantly reduce the chance of acquiring infection, early suggestions indicate that this could avoid onward transmission; this is especially relevant to transmission from asymptomatic healthcare workers to patients, but also in the other direction. ${ }^{13}$

An important and related point is that clinical acumen at an individual level, and clinical systems at an organisational level, must not disregard alternative clinical diagnoses and their appropriate management while screening for COVID-19.

\section{New ways of working: electronic consulting, remote working and doctor education at a distance}

Modern medicine is highly complex and commonly requires multidisciplinary team-working to plan and deliver patient care. Decision-making processes may have to be streamlined in order to utilise minimum staff and respect social distancing, and videoconferencing should be employed wherever possible.

In order to minimise time spent within the hospital, remote working should be enabled for clinical sessions where this is appropriate, with MDT meetings held via video conference and telephone clinics delivered from home, and administrative and SPA duties can often be performed remotely. All clinical work 
Table 1 summarises the various methods that may be used to reduce infection of staff and patients while delivering elective clinical services

\begin{tabular}{|c|c|}
\hline Concept & Example \\
\hline Hospital avoidance & $\begin{array}{l}\text { Clinic consultations by telephone or video call } \\
\text { Elective procedure consent by telephone or video call } \\
\text { Strict rules on permission for friends or relatives to join patient for hospital appointments } \\
\text { Hybrid care - specialist advice from hospital and hands on treatment in community } \\
\text { Decentralised care - physiotherapy, rehabilitation, delivered in community settings }\end{array}$ \\
\hline Patient separation & $\begin{array}{l}\text { Unidirectional flow with separate entrances and exits into hospital and clinic sites } \\
\text { Avoid mixing inpatients and outpatients } \\
\text { Provide elective services at different sites or times to inpatient services } \\
\text { Increased physical spacing in reception areas } \\
\text { Patients to wait outside while awaiting being called in to be seen for appointment } \\
\text { Drive-through services for pharmacy collections, pacemaker checks and other amenable } \\
\text { services }\end{array}$ \\
\hline $\begin{array}{l}\text { Pre-admission screening for } \\
\text { COVID-19 }\end{array}$ & $\begin{array}{l}\text { Questionnaire } \\
\text { Temperature check } \\
\text { Testing for active infection } \\
\text { Self-isolation } \\
\text { Possible role for immunological testing }\end{array}$ \\
\hline Infection control & $\begin{array}{l}\text { Meticulous hand hygiene by staff and patients } \\
\text { Frequent cleaning of clinical and non-clinical areas } \\
\text { Appropriate PPE } \\
\text { Sufficient access to PPE } \\
\text { Special protection for high-risk workers, eg enhanced PPE, alteration of clinical duties } \\
\text { Face masks for staff and patients } \\
\text { Frequent testing of asymptomatic staff }\end{array}$ \\
\hline $\begin{array}{l}\text { Electronic conferencing, remote } \\
\text { working and doctor education }\end{array}$ & $\begin{array}{l}\text { Encourage remote telephone/video conferencing to reduce mixing of staff where possible, eg } \\
\text { MDT, management meetings } \\
\text { Promote off-site working for administration, SPA duties, remote clinics } \\
\text { Enhance new ways of medical education including online tutorials, lectures, video tutorials, } \\
\text { simulation, virtual reality }\end{array}$ \\
\hline Alternative clinical pathways & $\begin{array}{l}\text { Consider diagnostic and treatment approaches with similar efficacy but lower infection } \\
\text { transmission }\end{array}$ \\
\hline
\end{tabular}

no longer resides within the hospital site, and the culture of performing all scheduled clinical activities within the boundaries of the healthcare building must be revised as we work in the new normal. The cost of investment in equipment will be rewarded with reduced non-essential clinician footfall within the hospital. There will be numerous lifestyle advantages to this as well, especially for those with caring responsibilities or particular health needs.

One cost of such measures is the impact upon opportunities for education and training. Novel ways of learning will be required to compensate for this, including online learning, simulators, virtual reality and the use of video-conferencing for both group discussions and tutorials. This will also require a respect for confidentiality and appropriate consent if consultations or procedures are recorded or broadcast for teaching purposes.

\section{Alternative clinical pathways}

Often, there are equivalent means for achieving a diagnostic or therapeutic outcome, yet each of these may confer a different level of infection risk. For example, ultrasonic and endoscopic procedures require prolonged proximity of practitioner and patient and may pose increased infection risk in comparison to CT, MRI or nuclear imaging modalities. Standard medical pathways and practice must adapt to consider such risks while balancing diagnostic accuracy, therapeutic efficacy, availability and waiting times. While it is not easy to increase MRI and CT capacity in the short-term, mobile diagnostic scanning facilities represent a short-term solution while infrastructure is developed in the longer term (Table 1).

\section{Conclusion}

Dramatic changes in the structure of the elective healthcare system are required to meet the ongoing COVID-19 challenge while also meeting non-COVID-19 healthcare needs. Bold and dynamic leadership will be required to ensure that they are operated safely and at adequate capacity compared to the pre-pandemic level. We anticipate potential implications for cost, availability and waiting times. It is important that clinicians, managers and politicians engage early in assessing the challenges to delivering non-COVID care and participate in the design and development of the modern and safe clinical and workflow solutions that they will be expected to deliver. This will require bold and innovative changes that look to the future, with unsubstantiated dogma being challenged and replaced with expert-led and, where possible, evidence-based approaches. 


\section{References}

1 Zhu N, Zhang D, Wang W et al. A novel coronavirus from patients with pneumonia in China, 2019. N Engl J Med 2020;382:727-33.

2 Rose MR, Hiltz, KA, Stephens RS, Hager DN. Novel viruses, old data, and basic principles : how to save lives and avoid harm amid the unknown. Lancet Respir 2020, in press (doi: 10.1016/S22132600(20)30236-8).

3 Task force COVID-19 del Dipartimento Malattie Infettive e Servizio di InformaticaIstituto Superiore di Sanità. Epidemia COVID-19. EpiCentro, 2020. www.epicentro.iss.it/coronavirus/bollettino/ Bollettino-sorveglianza-integrata-COVID-19_23-aprile-2020.pdf.

4 Mei H, Dong X, Wang Y, Tang L. Managing patients with cancer during the COVID-19 pandemic: frontline experience from Wuhan. Lancet Oncol 2020;21:634-6.

5 Arons MM, Hatfield KM, Reddy SC et al. Presymptomatic SARS-CoV-2 infections and transmission in a skilled nursing facility. N Engl J Med 2020;382:2081-90.

6 Long Q-X, Liu B-Z, Deng H-J et al. Antibody responses to SARS-CoV-2 in patients with COVID-19. Nat Med 2020;26:845-8.

7 Guo L, Ren L, Yang S et al. Profiling early humoral response to diagnose novel coronavirus disease (COVID-19). Clin Infect Dis 2020, in press (doi: 10.1093/cid/ciaa310).

8 NHS England. Operating framework for urgent and planned services in hospital settings during COVID-19. NHE, 2020. www.england.nhs.uk/coronavirus/wp-content/uploads/sites/ 52/2020/05/Operating-framework-for-urgent-and-plannedservices-within-hospitals.pdf.

9 Hellewell J, Abbott S, Gimma A et al. Feasibility of controlling COVID-19 outbreaks by isolation of cases and contacts. Lancet Glob Health 2020;8:e488-96.

10 Choi SC, Ki M. Estimating the reproductive number and the outbreak size of COVID-19 in Korea. Epidemiol Health 2020;42:e2020011.

11 Yuan J, Li M, Lv G, Lu ZK. Monitoring transmissibility and mortality of COVID-19 in Europe. Int J Infect Dis 2020;95:311-15.

12 Khalil A, Hill R, Ladhani S et al. COVID-19 screening of health-care workers in a London maternity hopsital. Lancet Infect Dis 2020, in press (doi: 10.1016/ S1473-3099(20)30403-5).

13 Klompas M, Morris CA, Sinclair ], Pearson M, Shenoy ES. Universal masking in hospitals in the Covid-19 Era. N Engl J Med 2020;382:e63.

Address for correspondence: Dr Sandeep S Hothi, Heart and Lung Centre, New Cross Hospital, Royal Wolverhampton NHS Trust, Wolverhampton WV10 0QP, UK.

Email: s.hothi@nhs.net 